
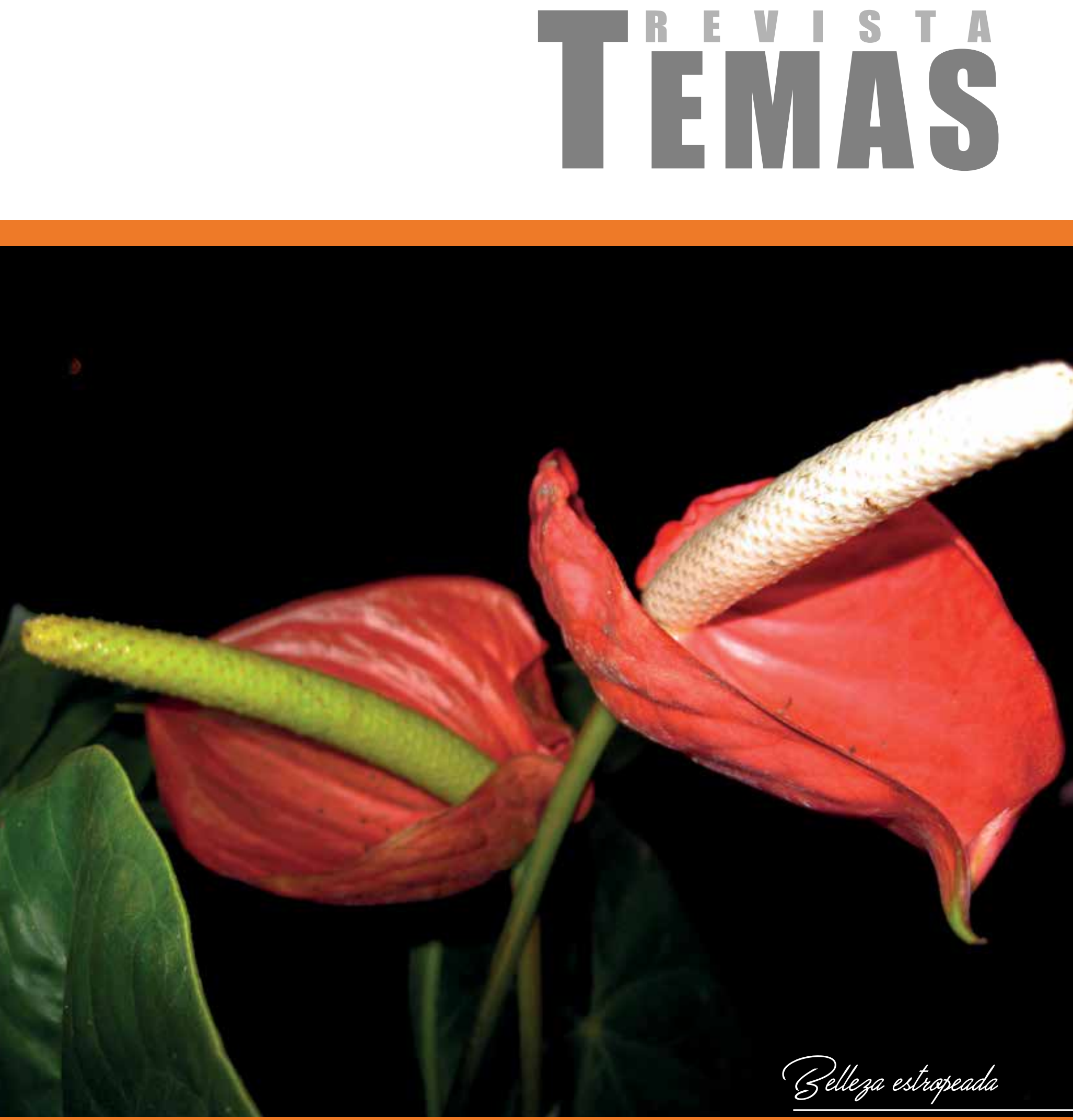

Referencia al citar este artículo:

Rodríguez, J. (2016). Biopoder y etnobotánica: experiencias del proyecto de aula "El saber de los abuelos" en la construcción de escenarios educativos para hacer las paces. REVISTA TEMAS, 3(10), 31 - 47.

\title{
Biopoder y etnobotánica: Experiencias del proyecto de aula "El saber de los abuelos" en la construcción de escenarios educativos para hacer las paces ${ }^{1}$
}

\author{
Jeyver Rodríguez Baños ${ }^{2}$
}

Recibido: 3/28/2016 Aceptado: 8/26/2016

\section{Resumen}

El artículo expone, desde el enfoque de la investigación-acción-participativa, la ruta seguida en la implementación del proyecto de aula "El saber de los abuelos". Este proyecto, orientado al rescate y revaloración de los conocimientos locales etnobotánicos buscó reflexionar sobre las implicaciones de la tecnociencia y el biopoder en las comunidades vivientes. El proyecto se estructuró desde el enfoque de las geopedagogías y la educación crítica como una propuesta capaz de promover en los estudiantes el desarrollo de capacidades investigativas relacionadas con el cuidado del medio ambiente, y el reconocimiento de los abuelos como portadores de saberes y prácticas de gran valor sociocultural. Al final del artículo se destacan los resultados de la salida pedagógica, la construcción de huertas caseras, la consulta de diversas fuentes de información, incluidos los herbarios digitales y la elaboración de fichas etnobotánicas como elementos dinamizadores del diálogo intercultural en la búsqueda de escenarios educativos para hacer las paces y propender por la preservación de la biodiversidad.

\section{Palabras clave}

Proyecto de aula etnobotánico, geopedagogías, diálogo intercultural, biopoder.

\section{Biopower and ethno-botany: Classroom project experiences of "the grandparents' knowledge" related to the construction of educational sceneries to make peace}

\begin{abstract}
Article exhibits, from the perspective of the participatory action research-, the path followed in the implementation of the project of classroom "Knowledge of the grandparents". This project, oriented to the rescue and local Ethnobotanical knowledge upgrading sought to reflect on the implications of the technoscience and BioPower in the living communities. The project is structured from the approach of them geopedagogias and it education critical as a proposed capable of promote in them students the development of capabilities research related with the care of the environment and the recognition of them grandparents, as carriers of knowledge and practices of great value socio-cultural. At the end of the article, include the results of educational output, the construction of home gardens, consultation of various sources of information, including the herbal digital and the elaboration of chips dynamic Ethnobotanical as elements of intercultural dialogue in the search for educational scenarios to make peace and promote the preservation of biodiversity.
\end{abstract}

\section{Keywords}

Project classroom ethnobotanical, geopedagogías, popular education, investigative skills, intercultural dialogue, biopower.

1 Artículo resultado de la investigación: “El proyecto de aula como estrategia didáctica para promover el desarrollo de competencias científicas y comunicativas en estudiantes de grados décimo y undécimo". Caso: Colegio público-rural de Puerto Parra, Santander. El proyecto se desarrolló en correspondencia con los objetivos de la Línea de Investigación en Didáctica de las Ciencias, bajo la dirección de la profesora María Helena Quijano, a quien agradezco el diálogo constructivo y sus aportes en todo el proceso de investigación. Por su aporte en el rescate de los conocimientos tradicionales etnobotánicos, la experiencia fue reconocida con mención al mérito pedagógico e investigativo en el Simposio Internacional de Educación y Pedagogía Crítica, Transformadora y por proyectos de vida, REDIPE. Cali, Colombia, 2013 y con mención al mérito otorgada por la Fundación Premio Compartir el Maestro 2013-2014.

2 Filósofo y magíster en Pedagogía. Doctorando Instituto de Filosofía. Becario VRI-CPD. Profesor adjunto de la Pontificia Universidad Católica de Chile. Correo electrónico: jrodriguez12@uc.cl 


\section{Introducción}

La etnobotánica escolar: entre el auge de los monocultivos y la extinción del mono araña café (Ateles hybridus)

La investigación se desarrolló en un colegio público rural del municipio de Puerto Parra, Santander, Colombia, en el cual se implementó el proyecto de aula "El Saber de los abuelos", diseñado desde la perspectiva etnobotánica. Este proyecto incluyó la construcción de huertas caseras y la consulta de herbarios digitales como una alternativa para recuperar los conocimientos de plantas medicinales entre la comunidad local. En su desarrollo, se utilizó la metodología etnobotánica (Bermúdez, Oliveira \& Velázquez, 2005), mediante la realización de entrevistas semiestructuradas; la elaboración de fichas etnobotánicas y la salida pedagógica para lograr el rescate de los conocimientos locales de las plantas medicinales. La revaloración de los conocimientos locales etnobotánicos cobró especial importancia en la investigación actual, contextualizada en la región del Carare, en la cual se está provocando un deterioro progresivo de la biodiversidad por el avance de los monocultivos de palma africana (Aricapa, 2007; Guerrero \& Wilches, 2015).

Figura. 1. Monocultivos de palma africana

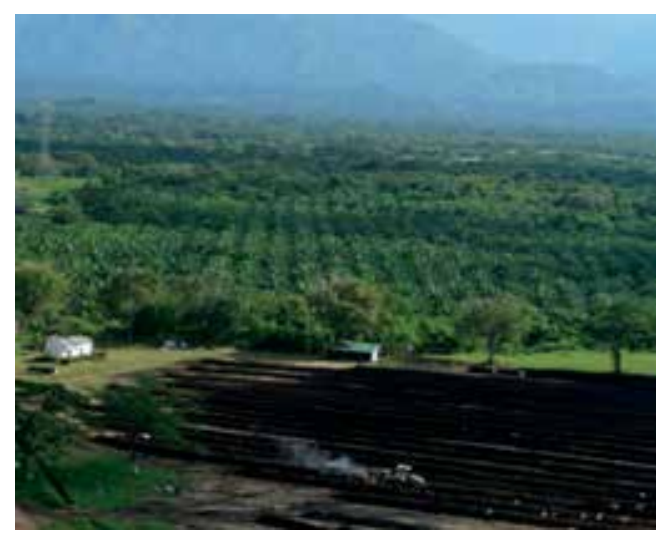

Fuente: Fedepalma.org, 2016.
Figura. 2. Foto de una hembra adulta y un macho subadulto de Ateles hybridus en la Serranía de Las Quinchas

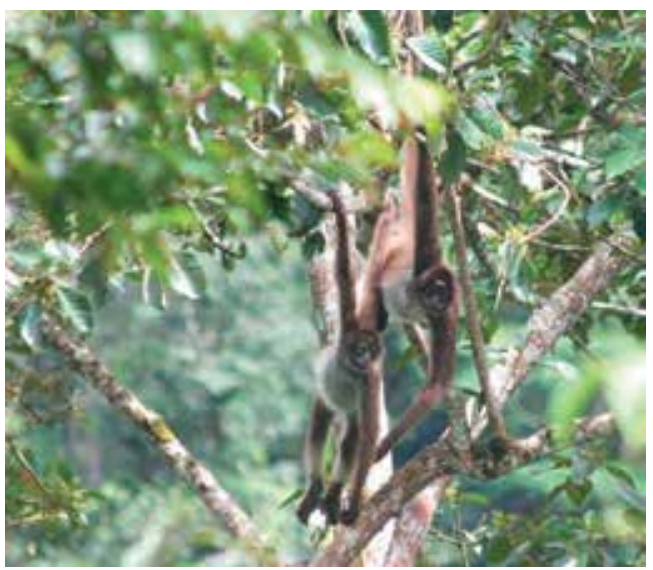

(Foto: Andrés Link).

Hasta ahora no se ha reflexionado sobre las consecuencias sociales y ecológicas de los monocultivos, al tiempo que se invisibiliza el daño causado en los caños y fuentes de agua y, por ende, en las comunidades de la zona. Las empresas dedicadas a la producción de aceite vegetal obtienen valiosos dividendos, ya que los cultivos no requieren altos costes de mantenimiento, pero si provocan gran contaminación debido al uso de pesticidas y fertilizantes (Ecologistasenaccion. org, 2011). En relación con el daño que están causando los monocultivos en los ecosistemas de la zona, Link, Luna y Burbano-Girón indican:

Los bosques húmedos tropicales del valle del río Magdalena en el piedemonte de Los Andes orientales han sufrido una dramática reducción, y actualmente el mosaico de fragmentos restantes está fuertemente amenazado por el establecimiento de monocultivos extensivos (ej.: palma de aceite) en esta parte del país. Sin embargo, esta región es también prioritaria para la conservación de Ateles hybridus, ya que contiene dos zonas con grandes extensiones de hábitat conservado: La Serranía de Las Quinchas en los departamentos de Boyacá y Santander y los bosques de la cuenca del río Opón en Santander (2013, p. 105). 
De lo anterior, nace la necesidad de crear un proyecto que llevará a la revaloración de los saberes etnobotánicos sobre los usos de las plantas medicinales, que sirviera de base para el empoderamiento social de los estudiantes. Este proyecto conceptuó la geopedagogía ${ }^{3}$, desde su relación con lo político, como un campo de saber y experiencia que exige redefinir las prácticas pedagógicas y la identidad del maestro desde los contextos de acción en los cuales se originó la experiencia pedagógica (Mejía, 2006).

En este sentido, se buscó construir sinergias entre actores (abuelos, portadores de saberes tradicionales etnobotánicos; estudiantes, portadores de explicaciones y conocimientos "alternativos" al discurso de la ciencia escolar y profesores, en su ejercicio de comprender y darle sentido a su práctica), generando nuevas formas de reconstruir las relaciones entre las comunidades locales y los ambientes, mediante la problematización de los conocimientos escolares y la co-construcción de nuevos saberes y prácticas que movilizaran el diálogo intercultural entre los adultos mayores y las nuevas generaciones, pues, de no existir este vínculo, muchos conocimientos locales de gran valor sociocultural y etnobotánico podrían caer en el olvido o desaparecer por el avance de la cultura tecno-científica (Olivé, 2006; Echeverría, 2010; Zent, 2001).

\section{Alfabetización científica y tecnociencia}

La alfabetización científica busca promover la toma de decisiones informadas

3 Estoy de acuerdo con Mejía (2011) cuando indica que "la geopedagogía no solo se refiere al contexto, sino a los territorios conceptuales, subjetivos, valóricos y de poder. Asistimos a un momento de creación de pedagogías, esa es una de las funciones fundamentales del maestro, la maestra y los educadores de este tiempo, que en educación pasan de ser portadores a productores de saber. En ese sentido, el ejercicio cotidiano del educador se hace profundamente político, en cuanto en él construye en el aquí $y$ en el ahora, sus horizontes de sociedad y de ser humano, $y$ al realizar el ejercicio de comprender su práctica en estos tiempos hace visible esa apuesta desde los enfoques y la metodología con la cual desarrolla su quehacer como educador" (pp. 158-159). sobre cuestiones que implican la cultura tecnocientífica y su impacto en la sociedad y los ambientes. Lo anterior cobra especial importancia en un contexto en el cual las comunidades locales, no solo quedan rezagadas de los beneficios de la cultura científica, sino que, además, deben sufrir las consecuencias nefastas que se derivan de la aplicación imprevisible y perjudicial de dicho conocimiento. Todo esto resulta manifiestamente insoslayable, en un momento en el que advertimos lo insostenible de nuestro actual sistema de producción y consumo:

un sistema socioeconómico guiado por la búsqueda del máximo beneficio particular a corto plazo, que apuesta por el crecimiento económico indefinido en un planeta finito, sin atender a sus consecuencias ambientales y sociales, lo que le convierte en responsable de los problemas que acabamos de enumerar y de otros igualmente graves, como las dramáticas migraciones, los conflictos y violencias causados por la competitividad, por el afán de controlar los recursos energéticos y otras materias primas esenciales y, en suma, por la destructiva anteposición de intereses particulares a la cooperación en beneficio de todos y de las generaciones futuras (Vilches $y$ Gil-Pérez, 2016).

La alfabetización científica exige reflexionar sobre las implicaciones de la tecnociencia en las comunidades vivientes, pues es allí donde cobran relevancia los problemas que tratan de enfrentar las ciencias naturales y sociales. En el proyecto de aula etnobotánico, se decidió por un énfasis pragmático de la enseñanza de las ciencias (Valladres, 2011), fundamentado en la resolución de problemas concretos, con lo cual se posibilitó transformar los programas y prácticas educativos hacia una mayor relevancia y pertinencia de los contenidos escolares ${ }^{4}$.

4 Vasen (2012) advierte que el biopoder da cabida a la popularización 
Con lo anterior, se buscó forjar un espacio de diálogo y cooperación interdisciplinaria en torno a las plantas medicinales, con vistas a promover el pensamiento crítico y una actitud investigadora en los estudiantes, "de tal manera que la vida se transforme en resistencia allí donde el poder asume como objeto la vida" (Foucault, 2005). Allí donde la vida se encuentra emplazada por lo político y, en especial, por la tecnociencia.

Se trata, como bien indica Agamben, del surgimiento de una nueva dimensión en la cual "ya no tendrá mucho sentido distinguir no sólo entre vida orgánica y vida animal, sino también entre vida biológica y vida contemplativa" (Agamben, 2007 , p. 91). Ese nuevo "tipo" de vida es, justamente, la que está siendo instrumentalizada por la tecnociencia: "las estrategias tecnocientíficas son subsidiarias del modelo de producción capitalista del que extrapola los saberes de y sobre la vida y que hace del saber biológico su principal instrumento de conocimiento y dominación; [...] la vida en las sociedades tardomodernas resulta afectada por las estrategias tecnocientíficas legitimadas por el discurso epistemológico científico oficial" (Lesteime, 2011, p. 43). En correspondencia con lo anterior, surge la siguiente pregunta: ¿Qué se entiende por y qué que exige la "alfabetización científica" para hacer frente a estos retos? El siguiente ejemplo de Olivé (2016) sirve para ilustrar lo anterior:

de una nueva "ética" semejante a una tabla valorativa de eficiencias ligadas al éxito y la inserción en el reino del consumo con lo cual la subjetividad comienza a ser entendida como objeto-fuente de aptitudes y skills que la posicionen como un bien vendible (2012, p. 145). En una dirección similar Mejía (2008) argumenta que con la emergencia de la biopolítica: Esas diferentes dimensiones de la vida: tiempo libre, cuerpo, son colocadas ahora en la esfera de la producción y el comercio no solo como bienes materiales e inmateriales, sino como formas de vida, comunicación, socialización. Y por ello, el proyecto de control se ve obligado a formar estándares de normatización para la educación, lo cognitivo, lo afectivo, organizando sistemas imperceptibles de ese control que son los que le dan forma a la biopolítica de estos tiempos. un campesino puede estar sembrando una semilla transgénica sin saberlo. Está sufriendo el impacto de la ciencia y la tecnología, y su cultura está siendo afectada también, pero eso no quiere decir que sea culto desde un punto de vista científico y tecnológico. Ser culto en ese sentido querría decir que sabe qué son las semillas transgénicas y que tiene la capacidad de decidir si las va a utilizar o no en función de su cultura y en ejercicio de su autonomía, sabiendo cuáles son los beneficios, los riesgos y los perjuicios que pueden tener los usos de determinadas tecnologías. Hablamos de una sociedad culta cuando hay personas que pueden decidir sobre qué tipo de tecnología se va a usar porque así les conviene y así lo deciden de manera autónoma.

\section{El proyecto de aula etnobotánico: “EI saber de los abuelos"}

El proyecto de aula etnobotánico se estructuró desde el enfoque de las geopedagogías y la educación popular (Mejía, 2006), como una propuesta capaz de auspiciar capacidades relacionadas con el cuidado del medio ambiente y el reconocimiento de los abuelos, quienes son portadores de saberes y prácticas de gran valor sociocultural relacionadas con los usos, preservación y cuidado de las plantas medicinales en el corregimiento Las Montoyas y sus zonas aledañas. En esta misma dirección, la UNESCO (2005) llama a valorar y proteger la diversidad cultural de los pueblos como un patrimonio común de la humanidad. $Y$ agrega:

la diversidad cultural crea un mundo rico y variado que acrecienta la gama de posibilidades y nutre las capacidades y los valores humanos, y constituye, por lo tanto, uno de los principales motores del desarrollo sostenible de las comunidades, los pueblos y las naciones.

El cuidado de la diversidad de la vida exige, por una parte, generar alternativas al desarrollo capaces de originar proyectos vitales que escapen a la lógica 
tecnocientífica que busca hacer predecible, controlable y medible la vida, y, por otra, optar por la construcción cooperativa de paces inacabadas, polisémicas y construidas desde diferentes ámbitos y con la participación de diversos actores. Se trata, en cualquier caso, de una "paz imperfecta" (Muñoz, 2007) capaz de rehacerse y de generar nuevas subjetividades y formas de resistencia que escapen al control y a la administración de la vida por el biopoder. En una dirección similar Fontan reclama reconceptualizar el enfoque de la paz desde una trama colonial puesta al servicio de las élites, apostando por una paz descolonizadora que

llama a una introspección de todos los aspectos de la industria de paz, a la trascendencia de la élite estructural hacia la formación y facilitación de procesos endógenos sostenibles comunitarios de paz. Ello trae parte de lo invisible al primer plano e involucra el desmantelamiento de las narraciones "oficiales" que reivindican a la primera persona y a las experiencias subjetivas de todos aquellos involucrados como visible y relevante (Fontan, 2012, p. 43).

Hoy enfrentamos graves problemas relacionados con la pérdida de la diversidad cultural; la violencia epistemológica (Pulido, 2009; De Sousa, 2009, Bolívar, 2009), ejercida a través de múltiples formas de saber y de poder que invalidan, marginalizan y silencian los saberes emergentes de las comunidades locales; la crisis de los valores ciudadanos y ecosociales (Hevia, 2009); el desequilibrio ambiental y el deterioro de las identidades locales, entre muchos otros. Ante estos apremiantes problemas, la alfabetización científica crítica, surge como posibilidad para reencausar la educación por el sendero de las necesidades humanas (MaxNeef, M., 1986).

Este trabajo se contextualizó en un horizonte en el cual la actividad tecnocientífica tiene repercusiones en los ambientes y las comunidades locales, poniendo en riesgo conocimientos ancestrales sobre las plantas medicinales de gran valor socio-cultural; en consecuencia, se halla inmersa en un contexto en el que los valores, imaginarios e identidades populares se vinculan con una visión particular de lo que es la ciencia, la tecnología y la cultura. El proyecto de plantas medicinales ayudó a problematizar estos aspectos desde el aula escolar, concebida como un escenario para el diálogo compartido (Edwards y Mercer, 1994), la investigación formativa y el desarrollo de la argumentación científica escolar (Candela, 1991; Cazden, 1991).

\section{Metodología}

Desde el enfoque de la investigación acción participativa (Mckernan, 1999; Carr y Kemmis, 1988), se cuestiona la visión del docente como reproductor del currículo y se recupera el rol del profesor desde su lugar en el acto formativo como creador de situaciones auténticas de aprendizaje (Díaz-Barriga, 2007) e investigador de sus propias prácticas pedagógicas (Stenhouse, 1998); es decir, como un profesional capaz de tratar su práctica como provisional y sujeta a la transformación. En el estudio realizado, la IAP se convierte en la base para promover el desarrollo de la autonomía de los estudiantes y el profesor (Freire, 2006), configurando nuevas trayectorias y sentidos que impulsan la experiencia pedagógica hacia nuevos rumbos.

\section{Contexto}

En el ciclo de IA que se describe a continuación, participaron estudiantes de décimo y undécimo grado del Colegio Departamental Las Montoyas de Puerto Parra, Santander. El municipio de Puerto Parra se encuentra ubicado al occidente del departamento de Santander, sobre la margen derecha del valle del río Magdalena y posee una extensión de 761,04 $\mathrm{km}^{2}$. En los últimos años el municipio ha incursionado en el cultivo de caucho y la 
palma africana, buscando una posición en estos renglones de la economía del país (Gobernación de Santander, 2006). Sin embargo, paralelo al desarrollo de los monocultivos se han ido deteriorando los ecosistemas de la zona: "Es el "desierto verde", como se le conoce; un desierto que en Colombia ya se ha extendido a 73 municipios de trece departamentos" (Aricapa, 2007). El medio de transporte típico de la región es la "moto-garrucha", uno de los más utilizados por los habitantes del municipio:

Figura 3. "Moto-garrucha"

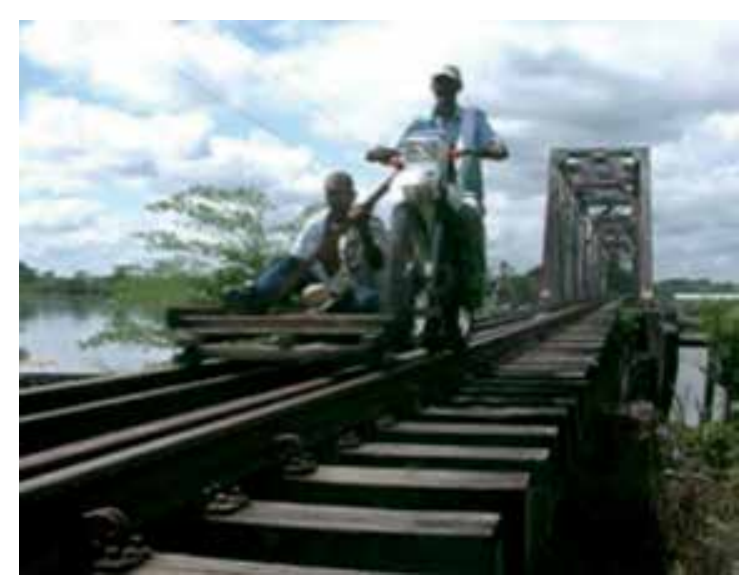

Fuente: Gobernación de Santander، 2006.

\section{Fases de la investigación}

El ciclo de investigación-acción se desarrolló en cuatro fases de la siguiente forma: En la primera fase, de exploración se definió el recurso al blog educativo como estrategia didáctica y el proyecto de aula como enfoque pedagógico. En la segunda fase, de diseño y planificación, se trazaron las estrategias didácticas orientadas a la acción formativa y al desarrollo de competencias científicas y comunicativas; para lograr lo anterior se trabajó conjuntamente con los estudiantes para determinar, desde sus intereses y necesidades, las preguntas del proyecto de aula, el cual se definió como un proyecto de plantas medicinales, desde la perspectiva etnobotánica. En la tercera fase se implementó el proyecto de aula sobre etnobotánica con la incorporación del edublog, teniendo en cuenta siete momentos que se detallan en la tabla 1 :

\section{Métodos y técnicas}

Se empleó la observación participante durante el ciclo de IAP, con el fin de examinar y documentar la actuación del maestro y los cambios en las actitudes de los educandos frente al proceso investigativo del proyecto de aula. Durante todo el proceso de investigación se recaudó información mediante notas de campo y grabaciones en audio de algunas sesiones de clases. Posteriormente, se realizaron dos grupos focales, con el propósito de profundizar en el proceso de acciónreflexión, dinamizado con la implementación del proyecto de aula desde la perspectiva de los educandos. Finalmente, se realizó la evaluación del proyecto de aula por los estudiantes, con adaptaciones al instrumento propuesto por (Mckernan, 1999), con el fin de recaudar datos sobre los aspectos claves del proyecto: relación profesor-estudiantes; pertinencia de los objetivos del proyecto; estrategias de aprendizaje; proceso evaluativo; acompañamiento del profesor; uso adecuado del lenguaje; entre otros aspectos. 
Notividades
No
1 Identificar los intereses científicos de los estu-
diantes.

2 Consulta de fuentes de información.

3 Reconocimiento del territorio.

4 Diálogo con "sabedores" de la región.

5 Procesamiento de datos.

6 Participación en la Semana de la Ciencia y el Emprendimiento.

7 Evaluación y socialización del proyecto.
Procesos de pensamiento y técnicas

Brainstorming, negociar y categorizar las ideas, plantear preguntas e hipótesis.

Consultar información en el blog, herbarios virtuales, páginas especializadas.

Salidas pedagógicas y elaboración de huertas caseras.

Entrevista semiestructurada.

Transcripción de entrevistas.

Elaboración de fichas etnobotánicas.

Elaborar productos medicinales.

Visita del Premio Compartir. Participación en Simposio de Pedagogía.

\section{Resultados}

Se describe la metodología utilizada en el proyecto de aula "El saber de los abuelos" y se problematizan algunos resultados que ayudan a configurar la práctica pedagógica como una experiencia escolar capaz de dinamizar las capacidades investigativas de los estudiantes. Teniendo en cuenta aportes de Lacueva (1998) y Ferreira y Peretti (2006), la estrategia didáctica se particularizó en la modalidad proyectos de aula investigativos o científicos, los cuales procuran que los estudiantes se apropien de conceptos, procedimientos y actitudes propias de la investigación escolar.

\section{Identificación de los intereses científicos de los estudiantes}

La identificación de los intereses científicos de los estudiantes en torno a las plantas medicinales se realizó empleando la técnica de "lluvia de ideas" o "brainstorming". Inicialmente, se planteó el problema como un desafío de conocimiento a partir de la pregunta: ¿Qué sabes a cerca de las plantas medicinales?

En relación con el uso del conocimiento científico, se observa que las ideas de los estudiantes, a partir del tópico propuesto, empiezan a desarrollarse de forma conexa en un proceso de construcción de sentidos y significados que permiten configurar el para qué del proyecto de aula, de forma tal que se supera lo unidireccional, configurando múltiples rutas de acción y reflexión. Respecto a las expectativas observadas en esta fase exploratoria los informantes mencionan que el proyecto de aula les "sirve" para aprender acerca de esas "maravillosas plantas" y manifiestan que quieren "expresar sus conocimientos" a otras personas "de la comunidad". La figura 4 ilustra lo anterior: 
Figura 4. Elaborando ideas acerca de las plantas medicinales y sus usos

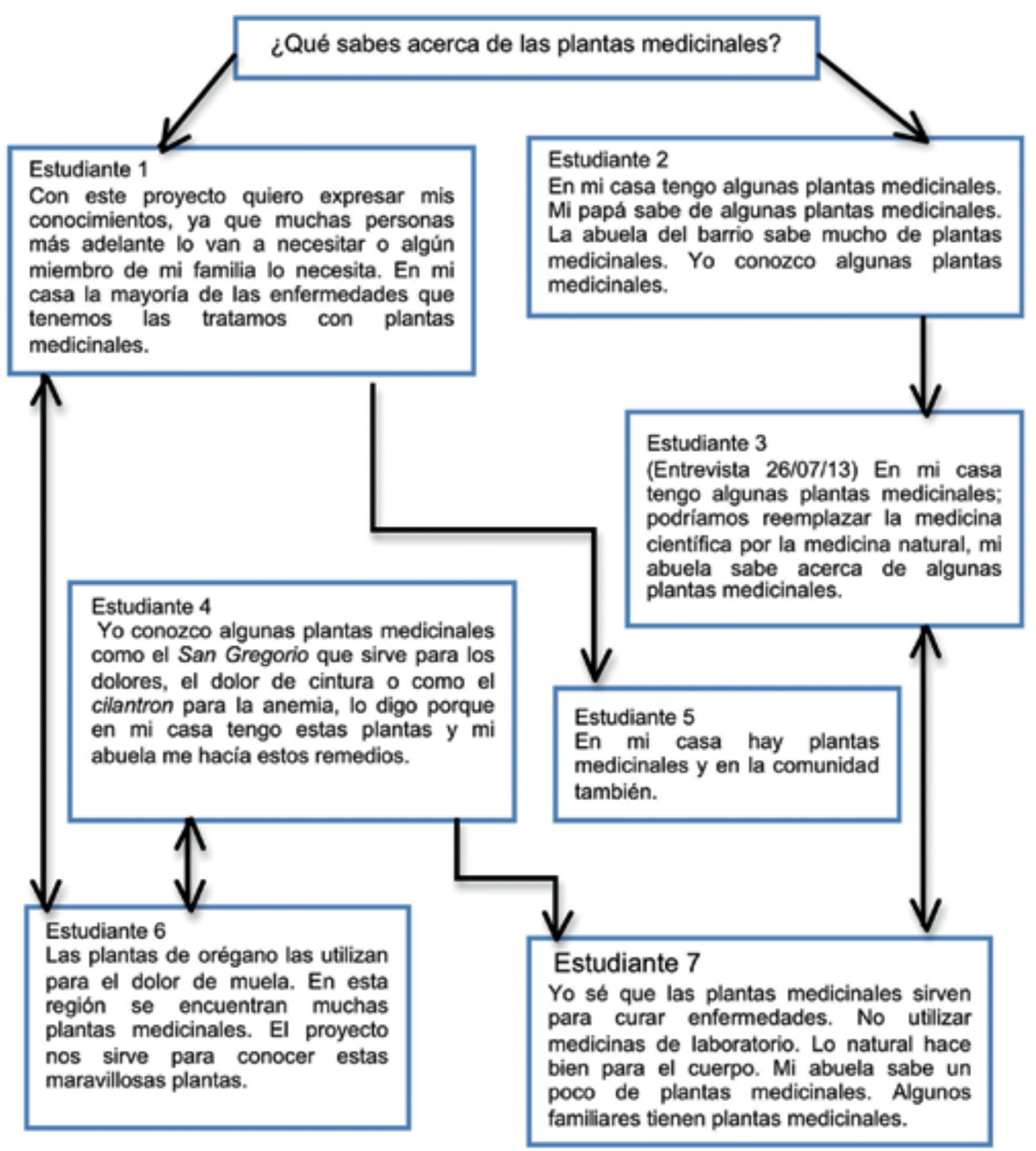

Fuente: Entrevista realizada a estudiantes de $11^{\circ}$, julio 17 de 2013

En esta fase, los estudiantes ponen a prueba la competencia referida al uso del conocimiento científico mediante la elaboración y transformación de ideas, lo cual exige del diálogo con otros estudiantes y con personas de la comunidad. De esta manera, el conocimiento se inscribe en el contexto local en diálogo con los imaginarios sociales que se construyen en torno a las plantas medicinales y sus usos. Al respecto, uno de los estudiantes resalta: "Las plantas medicinales son importantes porque no son pasadas por químicos o máquinas, son plantas naturales y seguras" (Entrevista, 26/07/13). Estas narrativas ponen en juego los conceptos de "salud" y "enfermedad" los cuales se contraponen a las nociones de "cura" y "remedio", propios de la cultura local. La experiencia pedagógica realizada corrobora la emergencia de conocimientos genuinos transdisplinarios que ayudan a generar sentidos y "líneas de fuga" que tensionan la práctica pedagógica. Así, se pudo constatar que los estudiantes, si bien de forma implícita, establecen 
relaciones complejas entre conceptos; proponen alternativas de solución a problemas relacionados con el uso de la "medicina científica" y relacionan el "conocimiento escolar" con los saberes y el conocimiento que emerge desde la comunidad.

Dentro de la dimensión de uso comprensivo del lenguaje científico, los sujetos implicados en esta investigación, relacionan la noción de "medicina occidental con "lo químico" y "lo perjudicial" para la salud, en tanto que el "conocimiento tradicional" de las plantas medicinales lo conciben como "natural y seguro" y como una "riqueza" que pueden utilizar, pues se encuentra a su alcance bien sea "en la casa" o en la "comunidad". Estas ideas al tejer un conjunto de relaciones entre los seres humanos y las plantas permiten configurar el proyecto de aula desde la perspectiva etnobotánica.

El proceso de elaboración de ideas permite a los estudiantes hacer uso paralelo de procesos científicos y comunicativos: los estudiantes seleccionan, dentro de su "enciclopedia de saberes", la información idónea para dar respuesta a la pregunta planteada, y luego transforman dichas ideas en preguntas de investigación. Ambos procesos promueven el desarrollo de habilidades cognitivo-lingüísticas, pues las ideas se someten a examen, se socializan con el grupo y deben plantearse de forma adecuada atendiendo a las demandas de la situación comunicativa (Lomas, Osoro y Tusón, 1993). Este proceso se ilustra en la figura 5.

Preguntas formuladas por los estudiantes

Figura. 5. Intereses científicos de los estudiantes de grados $10^{\circ}$ y $11^{\circ}$.

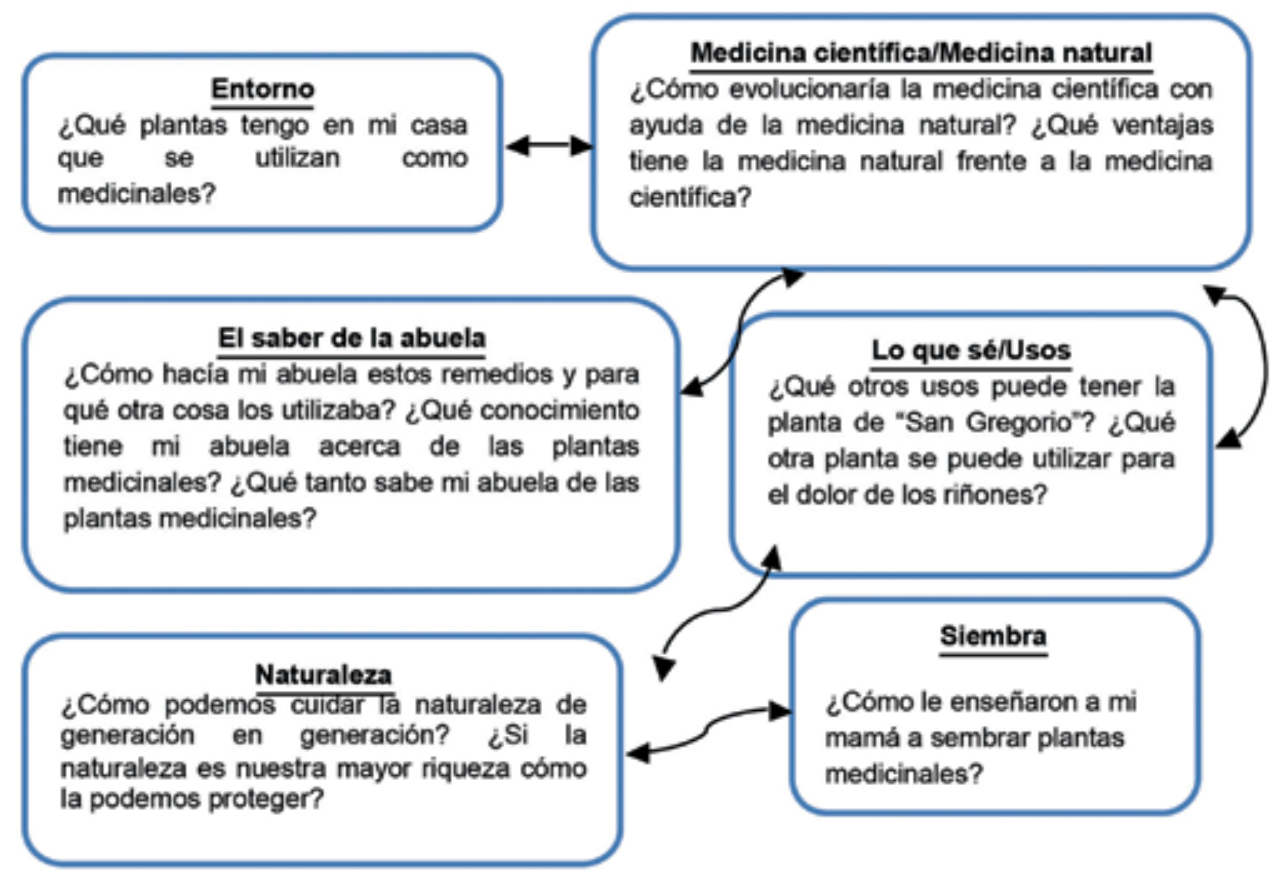

Fuente: Rodríguez, 2015.

La técnica "brainstorming" permitió hacer visibles los conocimientos e intereses de los estudiantes en torno a las plantas medicinales. Con ello se logró articular los intereses científicos de los estudiantes al proyecto, sirviendo como punto de partida de las primeras indagaciones. 


\section{Formulación de explicaciones alternativas}

Desde una concepción constructivista se asume que los estudiantes poseen sus propias explicaciones que provienen de sus particulares mundos de vida y que estas arrojan luz sobre el problema planteado. Por tanto,

aunque es cierto que estas ideas se contraponen o discrepan de la explicación científica, muchas de ellas no son "ilógicas", y en ocasiones están basadas en representaciones alternativas que cumplen una función útil en el procesamiento cotidiano de la información (Limón y Carretero, 1997).

El valor de la educación científica, de acuerdo con estos planteamientos, consiste en darles a los discentes la oportunidad de reunir estos conocimientos previos, analizarlos y hacerlos evolucionar a través de la indagación y la reflexividad (Freire, 2006).

La propuesta didáctica buscó promover actitudes científicas relacionadas con el interés por la actividad científica, la curiosidad, la perseverancia, la responsabilidad y el deseo de aprender. Ello se evidenció en el proceso de elaboración y formulación de hipótesis encaminadas a darle sentido a la problemática planteada sobre los usos de las plantas medicinales. La tabla 3 ilustra algunas hipótesis que sirvieron para orientar y determinar los procesos y actividades de aprendizaje dirigidas a dar solución al problema de investigación planteado en el marco del proyecto de aula "El saber de los abuelos":

En este proceso se promueve la competencia de explicación de fenómenos, la cual se relaciona con la manera en que los estudiantes van construyendo sus explicaciones en el contexto de la ciencia escolar. En estas explicaciones se van estableciendo relaciones entre las plan- tas, las costumbres y el dialecto propio de cada comunidad. Además, emerge la noción de identificación la cual es una categoría propia del trabajo etnobotánico.

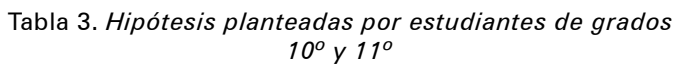

¿Por qué los nombres de las plantas medicinales varían de una población a otra?

“Todo varía dependiendo de la población donde se encuentre la planta y dichas costumbre del lugar"

"Porque cada población tiene costumbres diferentes y un dialecto diferente"

“Por el lenguaje que pueda tener la comunidad"

“Por el conocimiento que cada poblador tiene de ella, así es el nombre con el que se le conoce y es más fácil identificarla, mientras que por ejemplo en otras poblaciones las conozcan por su tamaño, así la identificaran"

Fuente: Rodríguez, 2015

\section{El edublog "El saber de los abuelos" como recurso mediador del aprendizaje}

En la práctica pedagógica se logró constatar que el blog educativo, utilizado como recurso mediador del aprendizaje, facilita el trabajo en equipo, promueve la consulta de artículos etnobotánicos de divulgación científica y la búsqueda en herbarios virtuales de los nombres científicos de las plantas identificadas como medicinales. Usar el blog de forma significativa, en el contexto del aprendizaje por proyectos de aula, implica colaborar con el aprendizaje de los demás, compartir lo que se sabe y hacer público qué se piensa. Sin embargo, uno de los aspectos que señalan los estudiantes como un obstáculo en el proceso de indagación es: "No tener los materiales como computador y acceso a internet para entrar al blog", pues esto hace que se pierda el "optimismo en el proyecto" (transcripción 
de grabación en audio, Autoevaluación, $\left.7 / 08 / 2013,10^{\circ}\right)$.

Progresivamente los estudiantes lograron comprender la investigación escolar como un proceso que exige reflexividad, diálogo y apertura mental para afrontar las incertidumbres. El proyecto de aula ayudó a idear escenarios para la construcción de la paz desde la recuperación de la caricia esencial, el respeto mutuo y la escucha activa. Con el paso del tiempo, los estudiantes lograron empoderarse, transformando sus sentimientos de rabia, fracaso y frustración en elementos dinamizadores de un cambio personal y social que empezaba en la confianza en sí mismos, en sus propias potencialidades y recursos cognitivos, afectivos y actitudinales.
En la figura 5 se muestra las opiniones de los estudiantes, obtenidas mediante la triangulación de fuentes y actores. Los estudiantes 1 y 4 con sus observaciones dan cuenta de la necesidad de "trabajar más" y "convivir más" en grupo para aprender a desarrollar su capacidad comunicativa y destacan la "autoconfianza" y la "tolerancia" como elementos que posibilitan el diálogo en un escenario de aprendizaje compartido. Todo lo anterior ayudó a configurar el proyecto de aula como un escenario en el cual los estudiantes pudieron concebirse no solo como miembros de una comunidad de aprendizaje sino, fundamentalmente, como sujeto crítico capaz de transformar y aportar ideas con visión a la solución de problemas relevantes de su entorno.

Figura 6. Relación entre actos comunicativos y actitudes de trabajo en equipo

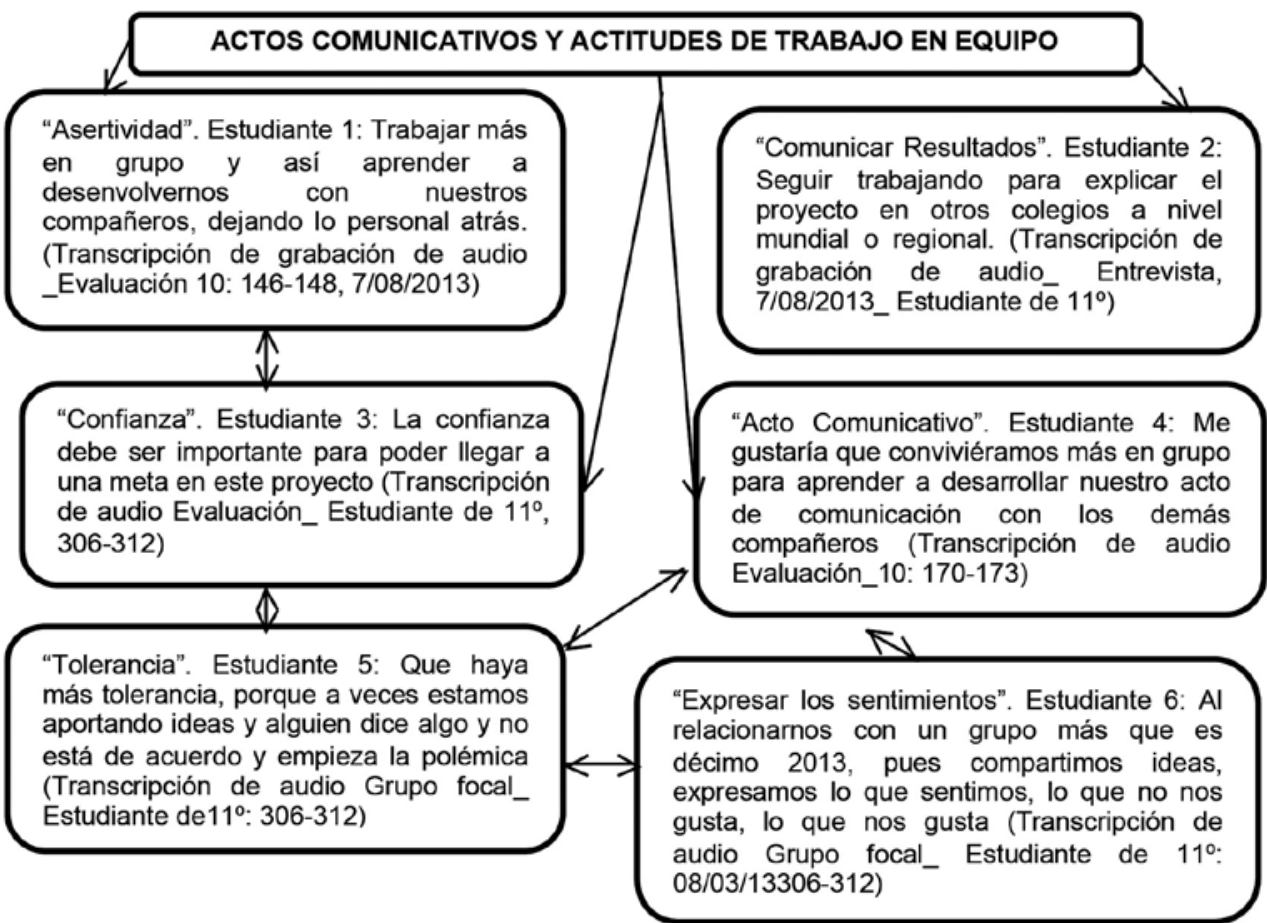

Fuente: Autor con datos de la Trascripción de evaluación y Grupo focal - estudiantes de $10^{\circ}$ y $11^{\circ}, 2013$.

\section{La salida pedagógica: una alternativa para la educación geográfica}

En la salida pedagógica los estudiantes aprendieron de la experiencia local con huertas caseras adelantada en la Ciénaga de Chucuri: el trabajo consistió en la recopilación de información sobre plantas medicinales a través de diarios de campo y realización de entrevistas a los 
pobladores de la ciénaga. Esta estrategia pedagógica promovió habilidades de investigación relacionadas con formulación de preguntas, la exploración del entorno, la observación, el trabajo en equipo y la transferencia de habilidades y recursos hacia otros contextos. Como resultado de la investigación realizada se logra definir la salida pedagógica como una

actividad significativa en la enseñanza y aprendizaje de la geografía, puesto que permite desarrollar la capacidad de organización espacial de los estudiantes, al establecer relaciones entre el territorio físico y las funciones sociales que tienen lugar sobre este, potencia habilidades de pensamiento como la observación, la descripción y explicación del fenómeno geográfico (Rodríguez y Pérez, 2000).

En la figura 6 se sintetizan los comentarios recogidos en el edublog en relación con la salida pedagógica:

Figura 7. Comentarios de salida pedagógica

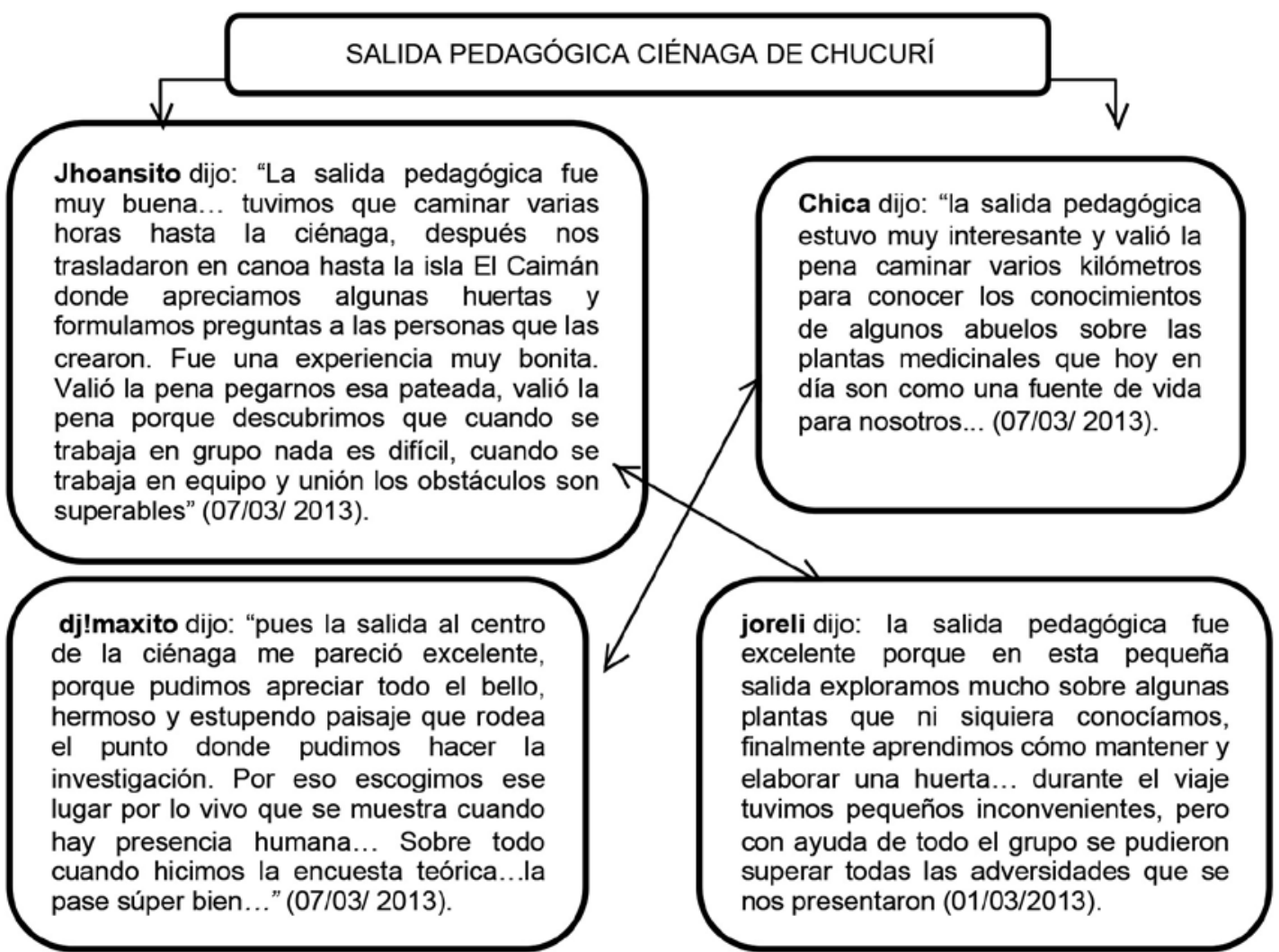

Fuente: Archivo Blog educativo, 2012

\section{La huerta casera y su aporte a la etnobotánica de la región}

En un momento posterior a la visita a la ciénaga de Chucurí y con los aprendizajes obtenidos a partir de la exploración del contexto, se implementó la huerta casera como una forma de recuperar el conocimiento tradicional de las plantas medicinales entre los pobladores del corregimiento Las Montoyas y sus zonas aledañas. La sistematización de la experiencia ayudó a caracterizar la cons- 
trucción de la huerta casera como una experiencia investigativa, desarrollada en el contexto del proyecto de aula, en el que los estudiantes formulan preguntas relacionadas con el tipo de suelo, las plantas, su mantenimiento y desarrollo evolutivo, las áreas aptas para la siembra, entre otros aspectos. Con el grupo de estudiantes se definió que solo se sembrarían plantas medicinales y en especial aquellas que los abuelos identificaron como muy significativas en la etnobotánica de la región. La figura 8 ilustra el trabajo realizado en la huerta.

Figura 8. Proceso de elaboración de una huerta casera en el corregimiento Las Montoyas

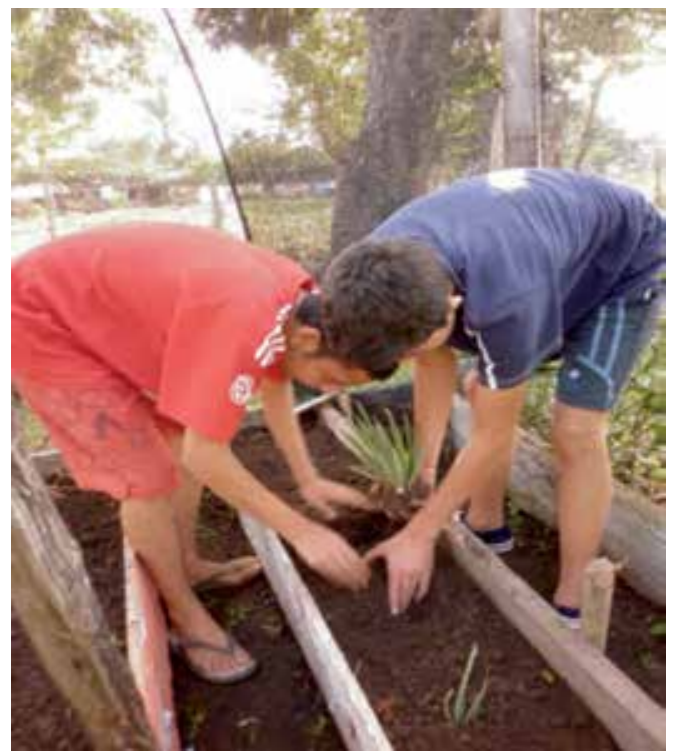

Fuente: Archivo fotográfico del blog educativo "El saber de los abuelos", 2013.

\section{Entrevistas a informantes claves: el levantamiento etnobotánico}

Para la realización de las entrevistas a informantes reconocidos en la región como "sabedores de las plantas medicinales", se utilizó el instrumento elaborado por (Fonnegra y Villa, 2011), el cual fue de gran utilidad al momento de realizar las entrevistas a 20 informantes claves, hombres y mujeres, en su mayoría mayores de 60 años, quienes suministraron información relacionada con el uso y aprovechamiento de las plantas medicinales en el corregimiento Las Montoyas y zonas aledañas al municipio de Puerto Parra, Santander. La figura 8 muestra a dos estudiantes quienes entrevistaron a la señora Petrona, reconocida por sus saberes sobre los usos de las plantas medicinales en la Ciénaga de Chucurí:

Figura. 9. Trabajo de campo realizado por dos estudiantes de $11^{\circ}$

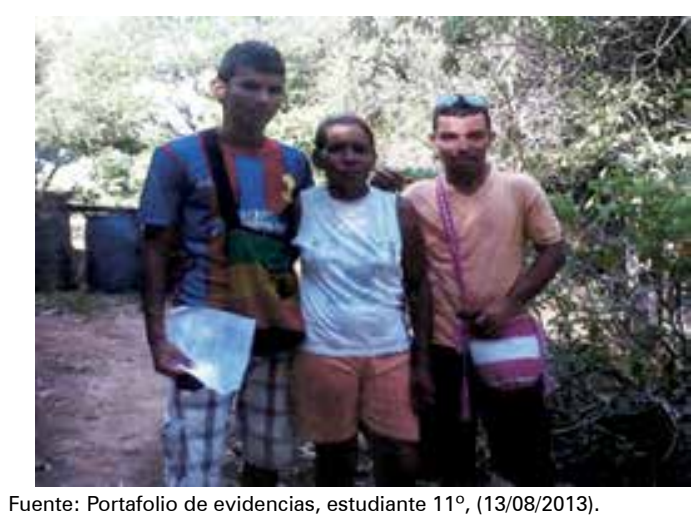

Estas entrevistas fueron grabadas en audio y posteriormente se transcribieron las partes significativas. En relación con lo anterior, uno de los estudiantes comentó en la evaluación final del proyecto: Un aspecto para mejorar es "haber hecho más entrevistas y compararlas unas con otras" (Autoevaluación_11: 55-56).

Esta consideración permite deducir que los estudiantes logran percibir el proceso investigativo como un todo en el que la información recolectada se debe contrastar para intentar hallar respuesta a las preguntas de investigación planteadas. Una vez recogida la información de campo, mediante entrevistas individuales a informantes clave, en su mayoría adultos mayores, los estudiantes, con ayuda de las profesoras de Cálculo e Informática, elaboraron tablas en Exce/ y organizaron la información etnobotánica recolectada. En la tabla 4 se muestran algunas plantas que son reconocidas por los sabedores locales como medicinales: 
Tabla 4. Algunas plantas medicinales reconocidas por Darío Yarse en Bocas del Carare Municipio: Puerto Parra, Santander, septiembre 2013

\begin{tabular}{|c|c|c|c|c|}
\hline Nombre común de la planta & Nombre científico & Parte utilizada & Preparación & Uso \\
\hline Altamisa & Artemisa Vulgaris & Hojas & Bebida & Cólicos \\
\hline Matarratón & Boccocina Frutescens & Hojas & Baños & Varicela \\
\hline Apio & Apium & Hojas & Bebida & Nervios \\
\hline Limoncillo & Litos Limonum & Hojas & Bebida & Gripa \\
\hline Silantrón & Coriandrum & Toda la planta & Bebida & Hepatitis \\
\hline Sábila & Aloe Vera & Cristal & Bebida & Gripa \\
\hline Orégano & Origanum Vulgare & Hojas & Soasada & Dolor de oído \\
\hline Árnica & Heterotbeca & Ramas & Baños & Desinflamación \\
\hline Pronto alivio & Lippia Alba & Hojas & Bebida & Dolores \\
\hline Paico & $\begin{array}{l}\text { Chenopodium } \\
\text { Ambrosioides }\end{array}$ & Hojas & Bebida & Nervios \\
\hline Cola de alacrán & Heliotropium & Cogollos & Baños & Infecciones \\
\hline Lengua de suegra & Sansevieria & Hojas & Bebida & Dolores \\
\hline Hierbabuena & Mentha Spicata & Hojas & Bebida & Cólicos \\
\hline
\end{tabular}

Elaborada por estudiantes de $11^{\circ}$. Fuente: Portafolio de evidencias de estudiantes de $11^{\circ}, 2013$.

De esta manera y como resultados del trabajo realizado, se hicieron visibles un conjunto de prácticas y saberes emergentes de gran valor sociocultural que brotan de las relaciones que se establecen entre el conocimiento escolar y los saberes etnobotánicos de la región. Lo anterior, cobró especial importancia en la investigación actual, toda vez que conecta con la construcción de una ciudadanía planetaria que se forja a partir del diálogo intercultural, bajo la premisa de que solo mediante el reconocimiento de la interdependencia entre los saberes tradicionales y los conocimientos científicos se podrá favorecer la preservación de la biodiversidad y los derechos de las comunidades locales como una forma de asegurar acercamientos holísticos a una realidad compleja.

Los resultados obtenidos en el proyecto de aula se presentaron en la V Semana de la Ciencia y el Emprendimiento, realizada del 30 de octubre al 1 de noviembre de 2013, en el colegio Departamental Las Montoyas. 


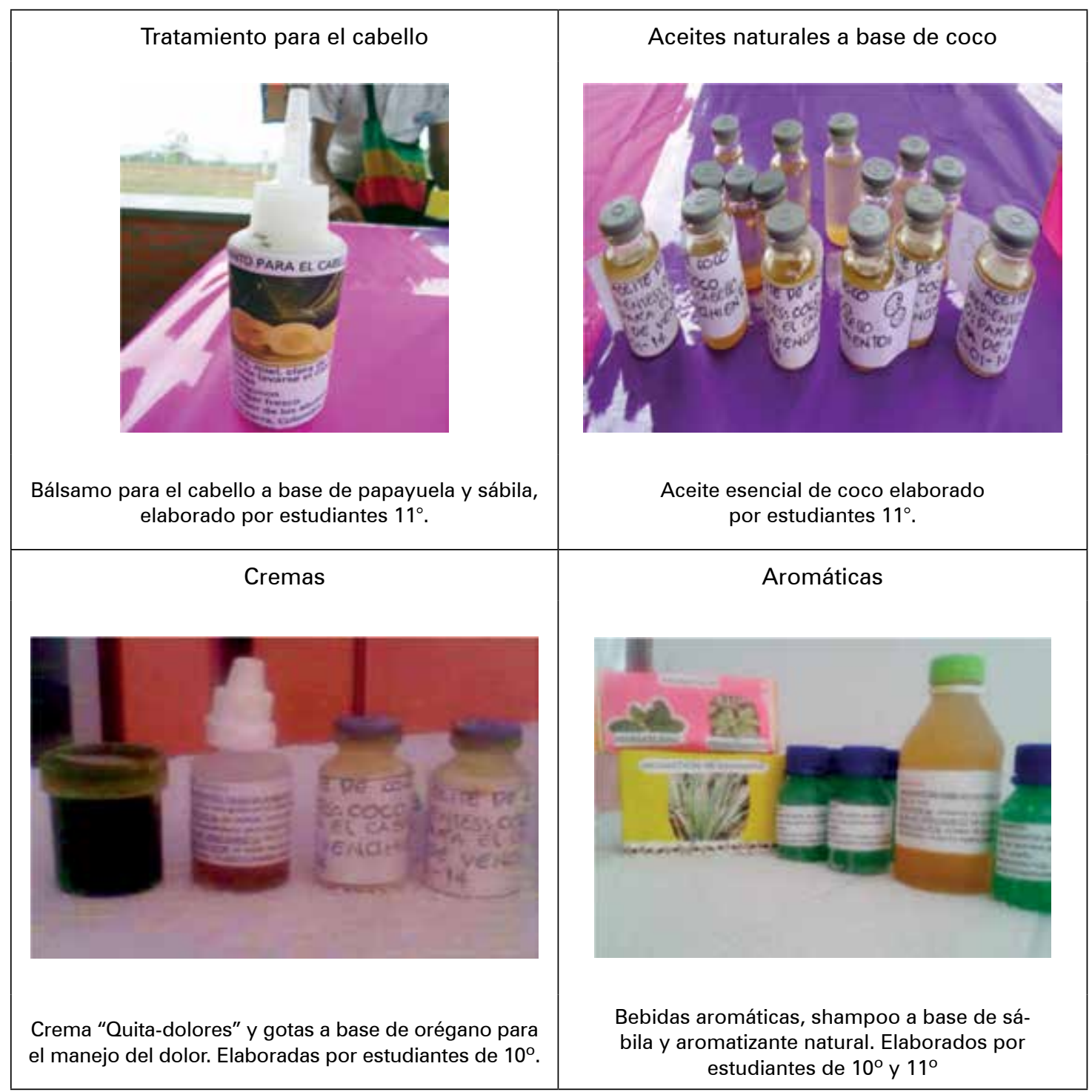

Fuente: Archivo fotográfico Blog educativo, 2014.

Como resultados finales de la investigación, cabe destacar la amplia gama de plantas que fueron reconocidas por los pobladores como medicinales, lo cual permite caracterizar la estrategia didáctica del proyecto de aula, en el que se incorporó el blog educativo como un escenario dinamizador de las relaciones entre la comunidad, la familia, la escuela y el ambiente geográfico, llevando a la recuperación del conocimiento etnobotánico sobre los usos tradicionales de las plantas medicinales entre los miembros de la comunidad local.

\section{Socialización y divulgación de la experiencia}

Posteriormente a la Semana de la Ciencia, el proyecto de aula fue divulgado en el marco del "Simposio Internacional de Pedagogía Crítica, Transformadora y por Proyectos de vida" organizado por la Red Iberoamericana de Pedagogía-REDIPE y 
celebrado en Cali los días 7 y 8 de noviembre de 2013. El proyecto de aula “EI saber de los abuelos" fue exaltado con Mención de Honor al mérito pedagógico e investigativo, por reconocerse como experiencia que logró recuperar el saber de las plantas medicinales, promoviendo la formación de seres sensibles, críticos y emprendedores de proyectos y acciones para enfrentar los retos de la sociedad y participar en la construcción de mundos mejores.

\section{Conclusiones}

En el ciclo de IAP descrito, el proyecto de aula se concibió como una estrategia didáctica y como un enfoque pedagógico orientado a promover el desarrollo de competencias investigativas en los estudiantes. Sin embargo, el proyecto de aula no partió, como es habitual, de los contenidos de la ciencia escolar, antes bien, intentó habilitar los contextos de vida y acción de los estudiantes para articular sus intereses, necesidades y expectativas en el desarrollo del proyecto.

En consecuencia, las actividades científicas y comunicativas desarrolladas en cada una de las fases del proyecto no se concibieron a partir de los contenidos de las asignaturas, por el contrario, se estructuraron de cara a los problemas de contexto relacionados con los usos de las plantas medicinales. Así, el proyecto de aula buscó constituirse en una ruta educativa capaz de promover la acción social y la corresponsabilidad de los jóvenes frente al cuidado de los ambientes.

El proyecto de aula aportó a la construcción de escenarios educativos para hacer las paces con el territorio y los ambientes, mediante el diálogo intercultural con los "sabedores" de la comunidad. A lo largo del proyecto se forjó un tiempo compartido materializado en la espiritualidad del viaje (Zambrano, 2007), en la confianza de los unos y las otras, en el reconocimiento de la vulnerabilidad como factor decisivo en la búsqueda de una convivencia que nos permita solventar los conflictos de manera pacífica y negociada.

En este orden de ideas, la tarea pedagógica se revela a partir del diálogo de saberes, del encuentro pleno con la alteridad y en la confluencia de proyectos e iniciativas compartidas, en procesos de investigación-acción concebidos en clave de futuro. Esta orientación epistemológica permite superar la instrumentalización pedagógica que conlleva a la no reflexión de las actividades educativas. Lo anterior se logra en un diálogo genuino con las tradiciones ancestrales y con los saberes locales de los cuales son depositarios los jóvenes. Se trata, por tanto, de asumir el descubrimiento de una identidad terrenal anclada en una clara consciencia del nosotros y de nuestra pertenencia a un espacio vital e intersubjetivo y a una condición histórica y cultural determinadas.

\section{Referencias}

Agamben, G. (2007). La inmanencia absoluta. En Giorgi, G. \& Rodriguez, F. (Ed.) Ensayos sobre Biopolítica (59-92). Excesos de vida. Buenos Aires: Paidós.

Aricapa, R. (2007). Las cooperativas de trabajo asociado en la agroindustria de la palma africana: Desierto verde y degradación laboral. Medellín: Fondo Editorial ENS.

Bermúdez, A., Oliveira-Miranda, M., \& Velázquez, D. (2005). La investigación etnobotánica sobre plantas medicinales: una revisión de sus objetivos y enfoques actuales. Interciencia, 30(8), 453-459. Recuperado de http://www.redalyc.org/articulo. oa? id $=33910703$

Bolívar, E. (2009). Medicinas y encuentros heterogéneos: Una mirada hacia el conflicto y el diálogo de saberes. En Reyes, G. L. (Ed.). Diálogo de saberes: Plantas medicinales, salud y cosmovisiones ( $\mathrm{pp}$ 105-110). Bogotá: Arfo Editores.

Candela, M. A. (1991). Argumentación y conocimiento científico escolar. Infancia y Aprendizaje, 14(55), 13-28. doi:10.1080/02103702.1991.10822302

Carr, W. \& Kemmis, S. (1988). Teoría crítica de la enseñanza. La investigación-acción en la formación del profesorado. Barcelona: Martínez Roca. Recuperado de http://data.theeuropeanlibrary.org/ BibliographicResource/2000065056769

Cazden, C. (1991). El discurso en el aula. El lenguaje de la enseñanza y el aprendizaje. Barcelona: Paidós.

Claret-Zambrano, A., y Cepeda, M. (2012). Contexto, significado y diseño de proyectos de aula en la enseñanza de las ciencias naturales. Cali: Editorial Universidad del Valle. 
De Sousa, B. (2011). Epistemologías del Sur. Utopía y Praxis Latinoamericana. Revista Internacional de Filosofía Iberoamericana y Teoría Social, 16(54), 17-40. Recuperado de http://dialnet.unirioja.es/ servlet $/$ oaiart?codigo $=4231309$

Díaz-Barriga, F., Hernández, G. (2002). Estrategias docentes para un aprendizaje significativo. México: Mc Graw Hill.

Echeverría, J. (2010). Tecnociencia, tecnoética y tecnoaxiología. Revista Colombiana de Bioética, Retrieved from http://www.redalyc.org/articulo. oa?id=189217244009

Edwards, D., y Mercer, H. (1994). El conocimiento compartido: El desarrollo de la comprensión en el aula. Barcelona: Paidós-MEC.

Elizalde, A. (2009). ¿Qué desarrollo puede llamarse sostenible en el siglo XXI?: La cuestión de los límites y las necesidades humanas. Revista de Educación, (1), 53-75. Recuperado de http://dialnet. unirioja.es/servlet/oaiart?codigo $=3019422$

Ferreyra, H., y Pereti, G. (2006). Diseñar y gestionar una educación auténtica: desarrollo de competencias en escuelas situadas. Buenos Aires: Noveduc.

Fonnegra-Gómez, R., \& Villa-Londoño, J. (2011). Plantas medicinales usadas en algunas veredas de municipios del altiplano del Oriente antioqueño, Colombia. Actualidades Biológicas, 33(95), 219250. Recuperado de http://search.proquest.com/ docview/1630026692

Fontan, V. (2012). Replanteando la epistemología de la paz: El caso de la descolonización de la paz. Perspectivas Internacionales, 8(1), 41-71. Recuperado de http://revistas.javerianacali.edu. co/index.php/perspectivasinternacionales/article/ viewFile/839/1364

Foucault, M. (2005). Historia de la sexualidad I: la voluntad de saber. Argentina: Siglo XXI.

Freire, P. (2006). Pedagogía de la autonomía: Saberes necesarios para la práctica educativa (11th ed.). Sao Paulo: Siglo XXI Editores.

Gobernación de Santander. (2006). Municipio de Puerto Parra Santander, esquema de ordenamiento territorial.

Guerrero, H., \& Wilches, J. (2015). Perspectivas multidimensionales de la paz en Colombia. Bogotá: Grupo Editorial Ibáñez.

Lacueva, A. (2004). La enseñanza por proyectos. Revista Iberoamericana de Educación, (16). Recuperado de http://rieoei.org/oeivirt/rie16a09.htm

Ledezma, A. L. P., \& Dueñas, W. A. M. (2016). Biopoder, desarrollo y alimentación en EI Rosal, Cauca (Colombia). Revista Colombiana de Sociología, 39(2).

Lesteime, D. (2011). Ciencia y biopoder: del discurso científico a la instrumentalización biopolítica del saber. Revista Colombiana de Filosofía de la Ciencia, 22(XI), pp. 47-55.

Limón, M., y Carretero, M. (1997). Las ideas previas de los alumnos. ¿Qué aporta este enfoque a la enseñanza de las ciencias? Buenos Aires: Aique.

Lomas, C., \& Osoro, A., y Tusón, A. (1993). Ciencias del lenguaje, competencia comunicativa y enseñanza de la lengua. Didáctica. Lengua y Literatura, vol 6, 272. Recuperado de http://revistas.ucm.es/index. php/DIDA/article/view/DIDA9494110272A

Link, Luna, y Burbano-Girón. (2013). Estado de conservación en Colombia de uno de los primates más amenazados con la extinción: el mono araña café (Ateles hybridus). Defler, T.R., Stevenson, P.R., Bueno, M.L., Guzman, D. (Eds.). Primates colombianos en peligro de extinción. Asociación Primatológica Colombiana, Bogotá.
Max-Neef, M., Elizalde, A., Hopenhayn, M., \& Herrera, F. (1986). Desarrollo a escala humana. Santiago: Centro de Alternativas de Desarrollo (CEPAUR).

McKernan, J. (1999). Investigación-acción y currículum ( $1^{\mathrm{a}}$ ed.). Madrid: Morata. Recuperado de http://data.the europeanlibrary.org/ BibliographicResource/2000066406133

Mejía, M. (2012). Educación(es) en la(s) globalización(es) l. Entre el uso técnico instrumental y las educomunicaciones ( $1^{\mathrm{a}}$ ed.). Bogotá: Desde Abajo.

Mejía, M. (enero-junio, 2011). Los movimientos educativos y pedagógicos del siglo XXI. En: Ciencia Política, 11, 128-156.

Mejía, R. (2008). Las pedagogías críticas en tiempos de capitalismo cognitivo. Ponencia presentada en el encuentro Maestros Gestores, Pedagogías Críticas y Resistencias. En Aletheia, 2(2), 22-24). Recuperado de http://aletheia.cinde.org.co/

Muñoz, F. (2007). Una paz compleja, conflictiva e imperfecta. Primer Seminario de Investigadores para la Paz de Andalucía, 1-23. Recuperado de http://www.ugr.es/ fmunoz/documentos/ pazcompconfimperfdraf.pdf

Olivé, L. (2006). Los desafíos de la sociedad del conocimiento: cultura científico-tecnológica diversidad cultural y exclusión. Revista Científica de Información y Comunicación, 3(3), 29-51. Recuperado de http://dialnet.unirioja.es/servlet/ oaiart?codigo $=2345507$

Olivé, L. (2016). Una cultura científica debe ser mucho más que el acceso a un teléfono móvil. [online] Recuperado de http://www.oei.es/historico/ divulgacioncientifica/entrevistas 092.htm [Accessed 16 Sep. 2016].

Pulido, G. (2009). Violencia epistémica y descolonización del conocimiento. Sociocriticism, XXIV(1-2) 173-201. Recuperado de http://sociocritica.org/ wp-content/uploads/2012/05/Sociocriticism-XXIV1-y-2-2009.pdf

Rodríguez, L., y Pérez de Sánchez, A. (2000). Ejercicio de la enseñanza de la geografía en las aulas escolares. Bogotá: Códice.

Stenhouse, L. (1988). La investigación como base de la enseñanza. Madrid: Morata.

Unesco. (2006). Convención sobre la protección y promoción de la diversidad de las expresiones culturales. Acción Pedagógica, 15(1), 136-148. Recuperado de http://dialnet.unirioja.es/servlet/ oaiart?codigo $=2968970$

Valladares, L. (2011). Las competencias en la educación científica: Tensiones desde el pragmatismo epistemológico. Perfiles Educativos, 132, (XXXIII) 159.

Vasen, J. (2012). Paradojas que habitan las instituciones educativas en tiempo de fluidez. En Taborda, Leoz, y Dueñas. (Comp.). (1 ${ }^{\text {a }}$ ed.). San Luis: Nueva Editorial Universitaria, U.N.S.L.

Vilches, Amparo, \& Gil-Pérez, Daniel. (2016). La Ciencia de la Sostenibilidad: una necesaria revolución científica. Ciência \& Éducação (Bauru), 22(1), 1-6. https://dx.doi.org/10.1590/15167313201600100017

Zambrano, A. (2007). Formación, experiencia y saber. Bogotá: Magisterio.

Zent, S. (2001). Acculturation and ethnobotanical knowledge loss among the piaroa of venezuela: Demonstration of a quantitative method for the empirical study of TEK change. In L. Maffi (Ed.), On biocultural diversity: Linking language, knowledge, and the environment. (pp. 190-211). New York: Smithsonian Institute. 